\title{
NGF Effects on Developing Forebrain Cholinergic Neurons are Regionally Specific*
}

\author{
Michael V. Johnston, ${ }^{1,6}$ J. Lynn Rutkowski, ${ }^{2}$ Bruce H. Wainer, ${ }^{3}$ Joseph B. Long, ${ }^{4}$ and \\ William C. Mobley ${ }^{5}$
}

(Accepted November 11, 1986)

\begin{abstract}
Nerve growth factor (NGF) has been shown to have an effect on neurons in the central nervous system (CNS). A number of observations suggest that NGF acts as a trophic factor for cholinergic neurons of the basal forebrain and the caudate-putamen. We sought to further characterize the CNS actions of NGF by examining its effect on choline acetyltransferase (ChAT) activity in the cell bodies and fibers of developing neurons of the septum and caudate-putamen. ChAT activity was increased after even a single NGF injection. Interestingly, the magnitude of the effect of multiple NGF injections suggested that repeated treatments may augment NGF actions on these neurons. The time-course of the response to NGF was followed after a single injection on postnatal day (PD) 2. NGF treatment produced long-lasting increases in ChAT activity in septum, hippocampus and caudate-putamen. The response in cell body regions (septum, caudate-putamen) was characterized by an initial lag period of approximately $24 \mathrm{hr}$, a rapid rise to maximum values, a plateau phase and a return to baseline. The response in hippocampus was delayed by $48 \mathrm{hr}$ relative to that in septum, indicating that NGF actions on ChAT were first registered in septal cell bodies. Finally, developmental events were shown to have a regionally specific influence on the response of neurons to NGF. For though the septal response to a single NGF injection was undiminished well into the third postnatal week, little or no response was detected in caudate-putamen at that time. In highlighting the potency and regional specificity of NGF effects, these observations provide additional, support for the hypothesis that NGF is a trophic factor for CNS cholinergic neurons.
\end{abstract}

KEY WORDS: NGF; cholinergic neurons; choline acetyltransferase development.

\section{INTRODUCTION}

The development of the nervous system appears to be critically dependent on the presence of soluble trophic factors $(1,2)$. One such factor is

${ }^{1}$ Departments of Pediatrics and Neurology, University of Michigan Medical School.

${ }^{2}$ Neurochemistry Laboratory, Department of Neurology, Johns Hopkins University School of Medicine.

${ }^{3}$ Committee on Neurobiology and Department of Pharmacological and Physiological Science, The University of Chicago.

${ }^{4}$ Department of Medical Neurosciences, Walter Reed Army Institute of Research. nerve growth factor (NGF), a protein molecule which has prominent, well-studied actions on developing sensory and sympathetic neurons of the peripheral nervous system (3-6). Recent data indicate that NGF also acts on neurons of the central nervous system (CNS). NGF increases the activity

${ }^{5}$ Departments of Neurology and Pediatrics, University of California School of Medicine, San Francisco.

${ }^{6}$ Address reprint request to: Dr. Michael V. Johnston, Neuroscience Laboratory Building, University of Michigan, Ann Arbor, Michigan 48104.

* Dedicated to Dr. E. M. Shooter and Dr. S. Varon as part of a special issue (Neurochemical Research, Vol. 12, No. 10, 1987). 
of choline acetyltransferase (ChAT), the synthetic enzyme for acetylcholine and a specific marker for cholinergic neurons, in immature rat brain tissue in vivo and in vitro (7-13). The NGF effect is specific for cholinergic neurons and their processes and is registered in the septum, nucleus basalis/substantia innominata, hippocampus, neocortex and caudateputamen $(8,10,12)$. The involvement of these neurons in processes which attend learning, memory $(14,15,16)$, and the control of movement $(17,18)$ has encouraged studies of the role played by NGF in the central nervous system (CNS).

Several lines of evidence suggest that NGF plays a physiological trophic role in the developing CNS. Rodent brain contains messenger RNA (mRNA) encoding NGF and the NGF protein (1922). Both NGF and NGF mRNA are enriched in areas with dense cholinergic innervation (e.g. hippocampus and neocortex) (20-22) and the neurochemical development of basal forebrain cholinergic cell bodies temporally coincides with the expression of NGF and its mRNA in these target areas (23). Moreover, receptors which are specific for NGF have been found in embryonic chick brain $(24,25)$, in the rodent brain on large, presumably cholinergic, neurons in the basal forebrain and caudate-putamen (26-28) and in human basal forebrain (29). NGF receptor molecules have also been detected in rodent hippocampus and neocortex (26, 27). NGF receptors appear to be localized on cholinergic neurites in these target regions for when radioactively-labelled NGF was injected into the hippocampus or cerebral cortex it was transported retrogradely to large basal forebrain neurons $(30,31)$. Finally, in recent experiments in which NGF antibody injections were timed to deprive developing neurons of their NGF supply, there were specific, long-lasting reductions in ChAT activity in the basal forebrain (32).

Studies of peripheral neurotrophic factors have demonstrated the potential importance of developmental stage in determining the magnitude and nature of their effects. For example, while fetal bovine adrenal medullary cells respond to NGF with neurite outgrowth and neutrotransmitter enzyme induction, neurites fail to grow from calf adrenal medullary cells and neither response is recorded with the adrenal medullary cells of adult animals (33). Developmental stage is also a critical determinant in the nature of the response of sensory and sympathetic neurons to NGF antibody injections. Antibody injections must be given at the appropriate developmental stage to destroy neurons (34). These observations suggest that there are stage-specific developmental alterations in the function and, perhaps, in the mechanism(s) by which NGF acts.

To further investigate NGF actions on cholinergic neurons of the forebrain, we have examined the effects of developmental stage on the ChAT activity response in septum, hippocampus and caudate-putamen. Using a number of injection protocols, we noted similarities as well as significant regional differences in the response of basal forebrain and caudate-putamen neurons to NGF administration. Of note, developmental stage had a clear effect on the ChAT activity response in the cauudate-putamen during early postnatal development.

\section{EXPERIMENTAL PROCEDURE}

NGF Preparation. Mouse NGF was prepared and characterized as described $(12,35)$. For some experiments, material was used which had been further purified by electrofocusing and gel filtration (12).

\section{Animal Preparation and Injection Technique}

Postnatal Animals. Pregnant Sprague-Dawley rats were individually housed and kept on a 12 hour daylight cycle. The birth of the litters was recorded every 12 hours and the day of birth was recorded as postnatal day (PD) 1. For early postnatal injections (i.e., PD2 through 8), a syringe needle (27 gauge; Hamilton) was placed approximately $2 \mathrm{~mm}$ anterior to the interaural line and 1.5 $\mathrm{mm}$ lateral to the sagittal suture and an intraventricular injection was made after direct transcutaneous puncture in restrained unanesthetized pups (10). For PD22 animals, anesthesia was achieved with Nembutal (20-25 mg/kg), the scalp was shaved, and a linear incision was made in the midline. After scraping the surface of the exposed bone, an injection was made with a 27 gauge needle approximately $2.5 \mathrm{~mm}$ lateral to the sagittal suture at a point $2 \mathrm{~mm}$ anterior to the intersection of the latter with the lambdoid suture and at a depth of approximately $3 \mathrm{~mm}$. Blue Dextran (Sigma) in phosphate-buffered saline was injected to confirm that injected material perfused the entire ventricular system.

Adult Animals. Intraventricular cannulae were placed in the right lateral ventricle in adult male rats $(225-250 \mathrm{gm})$ two to three days prior to NGF injection. After anesthesia with IM ketamine $\mathrm{HCl}(50 \mathrm{mg} / \mathrm{kg})$ and xylazine $(10 \mathrm{mg} / \mathrm{kg})$, the animals were prepared, as above, and a 27 gauge stainless steel cannula was sterotaxically implanted $8.7 \mathrm{~mm}$ anterior to the interaural line, 1.5 $\mathrm{mm}$ lateral to the sagittal suture and $4.0 \mathrm{~mm}$ deep to the surface of the skull. Injections were made via a saline filled PE 20 catheter attached to the cannula. Morphological investigation of cannula placement indicated that there was no injury to the septum, fimbria-fornix or hippocampus.

Intracaudate Injection. For intracaudate injection, PD22 animals were shaved, lightly etherized and transcutaneous puncture was made with a 27 gauge syringe. Coordinates for injection were established by prior test injections with cresyl violet. Accuracy 
of injection was established at sacrifice, $48 \mathrm{hr}$ later, by visually confirming the location of the injection track.

NGF Administration. A number of treatment schedules were used and these are specified in the text and legends. For either intraventricular or intracaudate NGF injections, $10 \mu \mathrm{l}$ of phosphate-buffered saline containing $30 \mu \mathrm{g}$ of NGF was administered. This NGF dose was shown in earlier studies to give large, reproducible changes in ChAT activity in basal forebrain neurons which were apparent within $48 \mathrm{hr}$ (12). Controls received an equal amount of cytochrome c (Sigma), a molecule physiochemically similar to NGF which does not have neurotrophic activity, or the vehicle alone. After each NGF or control injection in adult animals, the catheter was flushed with $4 \mu \mathrm{l}$ sterile saline and resealed by heating the tip. After injections, animals were returned to their home cages and rat pups were returned to the dam for the periods specified.

Brain Dissection and Enzyme Assay. Animals were killed by decapitation, the brains were quickly removed, divided sagittally and dissected on a chilled glass plate. The dissected regions included the septum, hippocampus, nucleus basalis/substantia innominata and caudate-putamen. The septum was dissected by disconnecting the hippocampus at the fornix and making two knife cuts immediately in front and in back of the septum continuing into the basal forebrain (Figure 1). The septum was then pinched away using curved forceps. The nucleus basalis/substantia innominata region was removed as follows: the rostral margin was the knife cut linking the root of the ventricle behind the septum to the base of the brain; another knife cut was then placed parallel to the first and 1.5 millimeters caudal to it; a piece of tissue, $2 \mathrm{~mm}$ thick, was removed which extended $1.5 \mathrm{~mm}$ dorsally from the base of the brain. To dissect the caudate-putamen, thalamic tissue was removed to the level of the internal capsule. Caudate-putamen was then taken anterior to the rostral margin of the internal capsule. In very young animals this was approximated by review of surrounding landmarks. To examine the cytological contents of the dissected areas in early postnatal animals, coronal and parasagittal sections were stained with cresyl violet. For detailed analyses in somewhat older (PD18-20) animals similar sections were prepared for immunocytochemical staining using a monoclonal antibody to $\operatorname{ChAT}(36,37)$. Rats were perfused intracardially with $20-40 \mathrm{ml}$ of cold saline, then with $150 \mathrm{ml}$ of a solution containing $2 \%$ paraformaldehyde and $.05 \%$

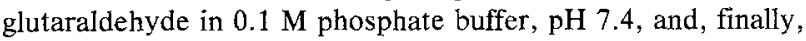
with $100 \mathrm{ml}$ of $10 \%$ sucrose in cold $0.1 \mathrm{M}$ phosphate buffer. The brains were removed, blocked, and cryoprotected in $30 \%$ sucrose in $0.1 \mathrm{M}$ phosphate buffer overnight at $4^{\circ}$. Frozen sections (50 $\mu \mathrm{m})$ were cut on a sliding microtome and submitted to immunostaining as described (36). Selected sections were counterstained with cresyl violet.

Brain homogenates were analyzed for the activity of ChAT (38) which was determined in relation to the concentration of soluble protein (39), as described (10). For measurements in developing animals entire litters were used and the overall ratio of males to females was approximately equal in the large groups used for these experiments.

\section{RESULTS}

Establishing a Method for Dissecting the Basal Forebrain and Caudate-Putamen. Landmarks for

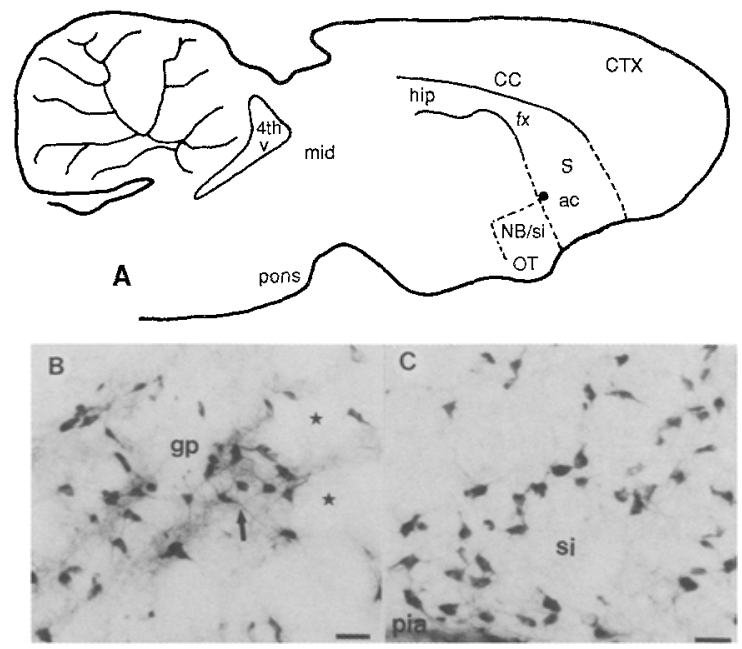

Fig. 1. Landmarks used for dissection of the rat brain for assay of ChAT. Panel A is a line drawing of a sagittal section through the brain of an adult rat; it illustrates the regions dissected. The area marked " $S$ " is the region identified in the text as septum and includes cholinergic parikarya in the medial septal nucleus, the vertical limb of the diagonal band of Broca and those in the most medial portion of the horizontal limb of the diagonal band. The method of septal dissection is given in the text. The hippocampus was separated from the septum at the fornix. The nucleus basalis/substantia innominata (NB/si) was dissected as described in the text. ChAT positive perikaryaya found in this region are shown in panels $B$ and $C$. Sections from 3 week old animals were immunohistochemically stained with a monoclonal antibody to ChAT. Panel B shows ChAT positive perikarya in the globus pallidus which are considered part of the nucleus bas. alis (37). The arrow points to ChAT positive neuronal processes and the stars indicate myelinated areas. Also included in the dissection were neurons of the substantia innominata as shown in Panel C. Other abbreviations: fx: fornix; ac: anterior commissure; th: thalamus; cc: corpus callosum; hip: hippocampus; mid: midbrain; 4th V: fourth ventricle; gp: globus pallidus; ot: optic tract.

brain dissection are considerably less distinct in the first two weeks of life than in the older brain. This is in part because myelination highlights important features of regional neuroanatomy. In order to ensure reproducible tissue sampling, a standardized dissection protocol was developed (Figure 1). Particular care was taken to separate the caudate-putamen, which contains a population of cholinergic interneurons, from the globus pallidus and substantia innominata, tissues which contain a separate population of cholinergic projection neurons (37). Figure 1 demonstrates the dissection landmarks and the morphologic characteristics of ChAT containing neurons in the globus pallidus and substantia innominata. As has been described, these basal forebrain cholinergic neurons are rather large and are intensely ChAT-immunoreactive. The fibers of cholinergic cells located in this, the nucleus basalis/ 
substantia innominata region, project to neocortex and amygdala (37). Cytologic preparations confirmed the location of these neurons in young animals and facilitated dissection of caudate-putamen. The septum contained cholinergic neurons of the medial septal nucleus and the vertical limb of the diagonal band of Broca, as well as cells in the most medial portion of the horizontal limb of the diagonal band. Neurons contained in the septal dissection constitute a more rostral collection of basal forebrain neurons which project to hippocampus (37); the latter was also taken. The dissection protocol thus made it possible to look at NGF effects on the cell bodies of basal forebrain cholinergic neurons and on their axons in target areas, as well as on the cell bodies and fibers of the cholinergic interneurons of the caudate-putamen.

Examining NGF Effects During the Development of Septo-Hippocampal Cholinergic Neurons. Initial reports of the in vivo response of central cholinergic neurons to NGF in immature rats used a series of injections during the early postnatal period with sacrifice on either the 8th or the 12th postnatal day $(8,10,12)$. The neurons of the basal forebrain and caudate-putamen undergo major developmental changes during early postnatal life. For example, ChAT activity in basal forebrain, hippocampus and neocortex increases markedly during the first three postnatal weeks $(23,40)$. Injection schedules which span wide developmental windows may obscure stage-specific developmental alterations in NGF response. In order to further examine NGF effects, we investigated the response to an abbreviated schedule of doses.

First, we examined ChAT activity on PD12 following NGF or control injection on: 1) PD2; 2) PD's 2 and 4; or 3) PD's 2, 4, and 6. The full schedule of 4 injections on PD's 2, 4, 6, and 8 elevated ChAT activity by $205 \%$ in the septum and $87 \%$ in the hippocampus $(10,12)$ (Figure 2$)$. Neither a single injection on PD2 alone, nor two injections on PD's 2 and 4 produced a detectable effect in the septum or hippocampus. Though 3 injections (PD's 2, 4, and 6) produced a $94 \%$ rise in the septum, no effect was registered in the hippocampus. These data suggested either that no NGF response was elicited by the earlier injections, or that the response to them was transient and disappeared by the 12th PD.

To determine whether the early NGF treatments had an effect, two abbreviated series of injections were carried out. Animals were injected on PD's 2 and 4 with assay on PD8 or were injected

\section{SEPTUM AND HIPPOCAMPUS}

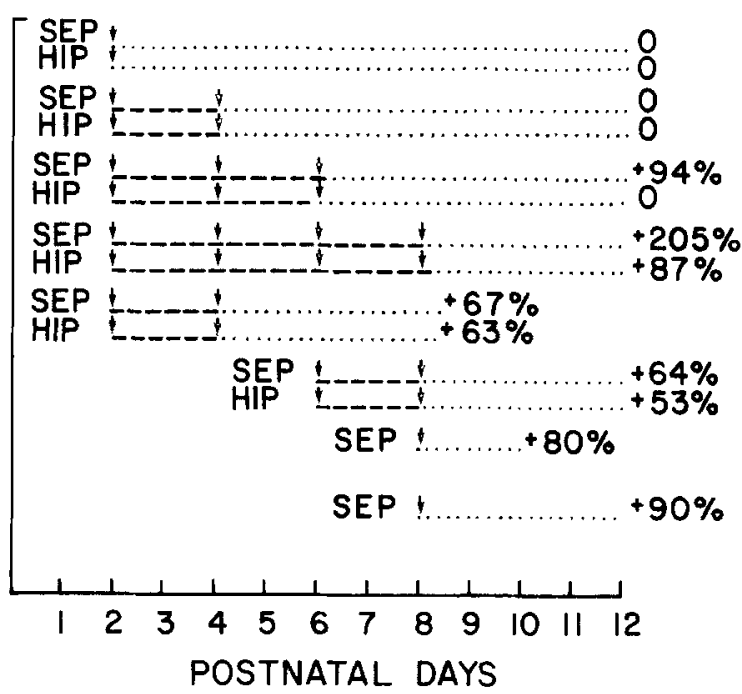

Fig. 2. Stimulation of ChAT activity in the septum and hippocampus by intraventricular injection of NGF on various postnatal days. The day of birth of rat pups was recorded as PD1. Litters of rat pups were divided into two groups of two to five pups which were then submitted to one of several different treatment schedules. Horizontal lines describe the effect of each of these different schedules on ChAT activity. NGF and control (vehicleinjected) groups were injected on the $\mathrm{PD}(\mathrm{s})$ indicated by the inverted arrows. For each NGF injection $30 \mu \mathrm{g}$ was administered. Details of the injection method are given in Experimental Procedures. Following the last injection, pups were returned to the dam for the period indicated by the dotted lines and the brains were dissected and assayed for ChAT activity. The numbers at the right indicate the mean percentage increase in ChAT specific activity in NGF-injected animals as compared with controls. Means only are shown for clarity. SEM's for the groups were $10 \%$ or less of the means. Where there was a mean increase in ChAT activity the increase was statistically significant at the $P$ $<.05$ level using Students' $t$ test.

on PD's 6 and 8 with assay on PD12 (Figure 2). Both schedules significantly increased ChAT activity in the septum and hippocampus. When expressed as percent increase relative to litter mate controls, NGF effects were equivalent in these two groups.

It was noted that no other series of injections produced the increases in ChAT activity seen with the initial series of four injections. Especially remarkable was the apparent discrepancy between the effect of 4 injections (PD's 2, 4, 6, and 8) and that recorded for two injections given on PD's 6 and 8 . A comparison shows that there was more than a three-fold difference ( $205 \%$ vs. $64 \%$ ) in the response in septum and about 1.8 -fold difference ( $87 \%$ vs. $53 \%$ ) in the response in hippocampus (Figure 2). Since the effect of injections on PD's 2 and 4 alone were no longer apparent by PD12 (Figure 2), it is 
not possible to ascribe the combined effect of 4 injections to simple addition of individual ChAT activity responses. Instead, it appears that the early NGF injections augmented the response to later administration.

An NGF effect on ChAT activity in septum was present beyond the early postnatal period. A single NGF injection $(30 \mu \mathrm{g})$ on PD22 elicited a $69 \%$ increase in ChAT activity within $48 \mathrm{hr}$ (PD24) (Figure 4). Indeed, a significant increase in ChAT activity was also registered in the septum of adult animals given multiple injections of NGF. Given every other day, a series of 4 injections increased ChAT activity in the septum of adult male animals by $36 \%$ relative to controls (inject $30 \mu \mathrm{g}$ NGF every other day for a total of 4 injections with sacrifice $24 \mathrm{hr}$ after the last treatment; mean $\pm \mathrm{SEM}$; control $=125 \pm 8$ nmol acetylcholine formed $/ \mathrm{h} / \mathrm{mg}$ protein $[n=6]$; $\mathrm{NGF}=170 \pm 6[n=6], 36 \%$ increase, $P<.01)$.

Interestingly, to date a ChAT activity response to NGF has not been detected in the intact hippocampus beyond the early postnatal period. Whether measured 48 or 96 hours after a single NGF injection at PD22, no response of hippocampal ChAT activity was apparent (inject $30 \mu \mathrm{g} \mathrm{NGF}$ on PD22 and sacrifice PD26; control $=90 \pm 12[n=3]$; NGF $=$ $95 \pm 5[n=5]$. The multiple NGF series described

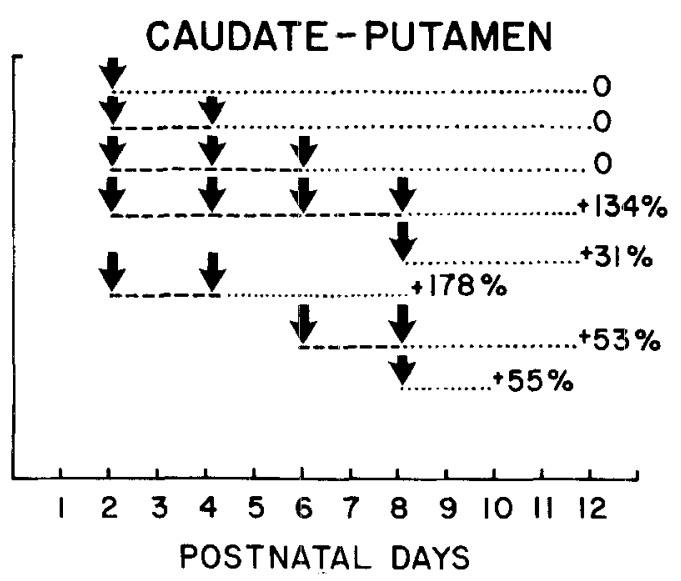

Fig. 3. Relationship between the timing of intraventricular injection of NGF and change in ChAT activity in the caudate-putamen of immature rats. Litters of rat pups were divided into two groups of two to five animals and submitted to NGF or control (vehicle) treatment as outlined in Figure 2. Following the last injection, pups were kept with the dam over the period indicated by the dotted lines and the caudate-putamen was then dissected and assayed for ChAT activity. The mean increase in ChAT specific activity in NGF-treated animals is shown by the numbers on the right. SEM's were $10 \%$ of mean. Mean increases in ChAT activity were significant at the $P<.05$ level.

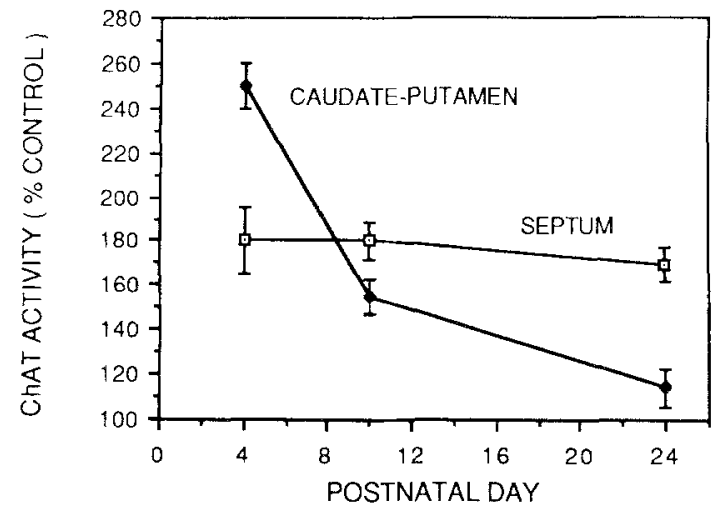

Fig. 4. Regional differences in the ChAT activity response to NGF injection during the development of forebrain cholinergic neurons. ChAT activity responses to single $(30 \mu \mathrm{g})$ NGF injections were determined $48 \mathrm{hr}$ after intraventricular administration. NGF-treated and control groups each consisted of 2 to 6 animals. Data for NGF-injected animals are given as percent specific activity of control (vehicle-injected) animals. Mean \pm SEM are listed over the postnatal day at time of assay.

for adult animals also failed to alter hippocampal ChAT activity (data not shown).

Examining NGF Effects in the Developing Caudate-Putamen. Using the same treatment protocols, the ChAT activity response to NGF was also examined in the developing caudate-putamen. Several features of the response were similar to those in septum. As was true in septum, injections confined to the first postnatal week were ineffective in increasing ChAT activity when assayed on PD12. Even 3 doses (PD's 2, 4, \& 6) failed to produce a response (Figure 3). However, an NGF response was clearly present in both of the two-injection series; this indicated that, like septum, the response in caudate-putamen was transient. Also, just as in septum, early NGF doses apparently augmented the response to later injections. Comparing the effect of 4 injections (PD's 2, 4, 6, \& 8) with two (PD's 6 $\& 8$ ), the effect in caudate-putamen was somewhat less pronounced than in septum, amounting to a 2.5fold ( $134 \%$ vs $53 \%$ ) increase (Figure 3 ).

Though similar to septum in these respects, there was a significant difference during development in the response of the caudate-putamen to NGF. While a series of two injections given on PD's 2 and 4 increased ChAT activity $178 \%$ relative to control, when delivered on PD's 6 and 8 NGF produced only a $53 \%$ increase (Figure 3 ). A decreasing responsiveness to exogenous NGF was equally apparent in the relative effectiveness of a single injection given on PD2 (155\% increase) or on PD8 
(55\%) (Figures 3, 4). Thus, there was a significant lessening of the NGF effect during this period. The differing responses in septum and caudate-putamen were even more marked in later developmental stages. Though a single injection of NGF at PD22 elicited a pronounced response in septum (see above), a small (15\%) and insignificant effect was recorded in the caudate-putamen (Figure 4). To determine whether failure of injected NGF to penetrate caudate-putamen was responsible for the lack of response, NGF was given directly into the caudate-putamen in 22 day-old animals. Animals were killed 48 hours after injection and the injection track was visually confirmed. These injections of NGF had no effect on ChAT activity (inject $30 \mu \mathrm{g}$ NGF on PD22 and assay on PD24; control $=650 \pm 50$ $[n=2] ; \mathrm{NGF}=674 \pm 60[n=3])$. The response of caudate-putamen in adult animals was not examined.

Time Course of NGF Effects in Cell Body and Fiber Regions of Early Postnatal Animals. To further examine the ChAT activity responses in septum and caudate-putamen, a single injection of NGF was given on PD2. There was no definite effect through the first 24 hours (Figure 5). Over the next 24 hours there was a brisk rise in septum (180\% of control) and caudate-putamen (260\%), which was maintained for at least an additional 48 hours. ChAT ac-

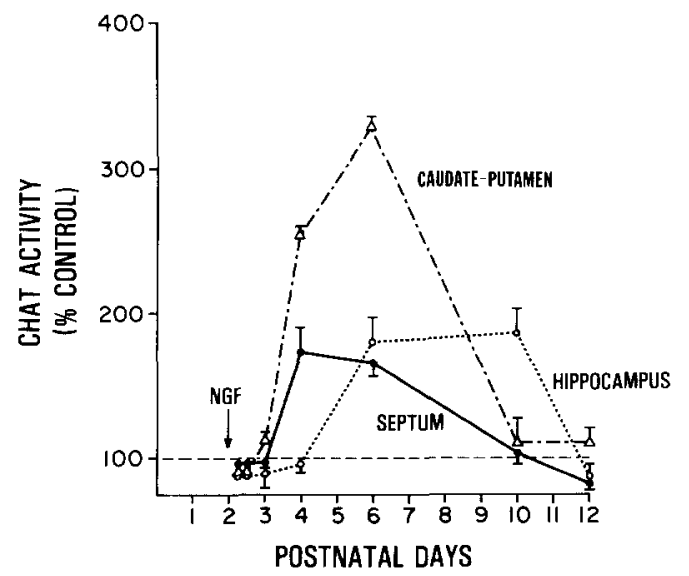

Fig. 5. Regional rise of ChAT activity in neonatal rat pups after a single intraventricular injection of NGF on PD2. Litters of rat pups were divided into groups of four or five and a single $(30 \mu \mathrm{g})$ injection of NGF was given on PD2. Following a specified postinjection interval, NGF-treated and control (vehicle-treated) groups were taken for assay of ChAT activity in the caudateputamen, septum and hippocampus. The elevation in ChAT specific activity in the NGF injected animals is shown as percent of control \pm SEM. Due to maturation, the absolute control value rose during the period over which these experiments were conducted. tivity returned to control levels by the PD10. The response in both these cholinergic cell-body regions was similar with respect to an initial lag period, a rapid rise to maximum values, a plateau phase and then a return to baseline.

The hippocampal response to NGF was distinct from that for septum. Most important, there was a clear lag relative to septum in both the ChAT activity increase and its subsequent decline. While a clear increase was present in the septum 48 hours after injection, hippocampus was unchanged at that time. Responses of the magnitude recorded in septum were first detected in hippocampus at 96 hours after injection and persisted through the 10th PD. The temporally distinct responses of septum and hippocampus indicated that the NGF effect on ChAT activity was first registered in developing cholinergic cell bodies.

\section{DISCUSSION}

As yet we know very little of the physiological principles or mechanisms which underlie neurotrophic interactions in the CNS. Nevertheless, current data for the regional distribution of NGF (2022 ) and its receptors (26) in the forebrain suggest that it is released in target areas to act on the neurites of developing and mature cholinergic neurons of the basal forebrain and caudate putamen. The present results demonstrate distinct regional differences in the timing and magnitude of responses to NGF and document that striking increases in ChAT activity result from even a single NGF injection. In highlighting the regional specificity and potency of NGF effects these observations provide additional support for the hypothesis that NGF has a trophic influence on CNS cholinergic neurons in septum and caudate-putamen.

The efforts taken in dissection made it possible to examine responses in brain regions containing different populations of cholinergic neurons and their fibers. Particular care was taken to separate the cholinergic neurons in the caudate-putamen from those in the basal forebrain found in nucleus basalis and substantia innominata. Using the dissection protocol, instances were observed in which the response of the nucleus basalis/substantia innominata was entirely different from that of the caudate-putamen. For example, following injections of NGF on PD's 2, 4, and 6, ChAT activity at PD12 was increased by $75 \%$ in the nucleus basalis/sub- 
stantia innominata (M. V. Johnston and W. Mobley, unpublished observation), but was unchanged in caudate-putamen (Figure 3). On the other hand, injections of NGF on PD's 2 and 4 produced a large increase in ChAT activity by PD8 in the caudateputamen (178\%) (Figure 3); the elevation in the nucleus basalis/substantia innominata was modest $(+24 \%)$ and similar to the responses recorded in the septum and hippocampus (Figure 2).

By examining responses to single injections it has been possible to observe NGF actions during relatively discrete periods during development. The ChAT activity response to a single dose in early postnatal life suggests that NGF is acting to transiently accelerate cholinergic neurochemical differentiation. After a single NGF injection on day 2, ChAT activity in the hippocampus remained at levels approximately twice those in littermate controls from PD6 to 10 (Figure 5). The normal ontogenetic pattern of ChAT specific activity in the hippocampus during this period demonstrates an extended plateau at about $50 \%$ of adult activity from seven to eleven days of age (40). Therefore, it can be calculated that a single NGF injection raised ChAT activity to near adult levels during this time. It would be of interest to determine whether these NGF effects are associated with other measures of neuronal differentiation. For example, the emergence of ChAT activity in the developing hippocampus has been correlated with cholinergic synapse formation $(40,41)$. It is possible that NGF-mediated increases in ChAT activity are associated with accelerated formation of cholinergic hippocampal synaptic contacts or with earlier onset of synaptic function; however, at present there is no proof of this. In view of the return of ChAT activity to baseline levels after NGF tretment, any effect of exogenous NGF on synaptogenesis may also be transient.

NGF effects were recorded in immature and mature septohippocampal cholinergic neurons. Responses were recorded as early as PD4 and in adult animals. Indeed, when expressed in terms of percent control, NGF-mediated increases in ChAT activity were as dramatic in PD22 animals as in younger ones (Figure 4). NGF effects were thus registered in developmental stages which precede the neurochemical differentiation of this projection and in those which follow the major period of synapse formation (42). In demonstrating a response in mature septo-hippocampal neurons, these data combine with measurement of NGF and its mRNA in adult hippocampus (20-23) to suggest that NGF may play a role in the maintenance of mature basal forebrain neurons. The nature of that role is not yet known but one corollary to these findings is suggested by NGF effects on sympathetic neurons. NGF injections in adult mice increased neurotransmitter enzyme activity in target regions in association with increased ramification of peripheral neurite networks (43). These data have been interpreted as reflecting an NGF-stimulated collateral sprouting of sympathetic neurites. It was speculated that endogenous NGF may play a similar role in maintaining mature sympathetic nerve networks (43). Similar events may describe NGF actions in the mature hippocampus. However, as noted, no ChAT activity response was detected in the hippocampus of NGFtreated rats beyond the early postnatal period. This disparity in septal and hippocampal responses is currently unexplained and suggests that developmentally determined events differentially influence NGF responses in cholinergic cell body and target regions. It is noteworthy that a ChAT activity response to NGF was seen in the hippocampus following transection of some cholinergic afferents (44) and that NGF treatment may enhance the sprouting of cholinergic fibers under such conditions (45). Yet to be determined is the relationship of these effects, and of the enhanced survival of lesioned septal cholinergic neurons which attends NGF treatment (46, 47) to NGF actions in intact, mature animals.

An unexpected observation in these studies was that early exposure to NGF augmented the response to later treatments. This effect was seen for both septum and caudate-putamen. In both regions, ChAT activity changes at PD12 were greater after 4 injections than after any shorter series assayed on the same day. In fact, when allowance was made for the expected fall in ChAT activity after early NGF injections, the effect of 4 injections was clearly greater than that which would have been achieved by simply adding the contributions of both 2-injection protocols. Thus, it appeared that early injections in the 4-injection protocol in some way altered the response of these tissues to later NGF administration. Several possibilities can be envisioned to explain these results. Earlier injections may have induced increased expression of NGF receptors or modified the events which transpire between NGFreceptor binding and the ChAT activity response. Regarding the latter, NGF effects on transcriptional or translational controls for ChAT may have "primed" cholinergic neurons for later injections. Further studies will be required to address the na- 
ture of the NGF-induced increases in ChAT activity.

NGF effects on cholinergic interneurons in the caudate-putamen were similar in many respects to those in the basal forebrain. However, there was a clear difference in the responses of septum and caudate-putamen to NGF delivered during later developmental stages. While septum continued to respond beyond the third postnatal week, a noticeable decline in the caudate-putamen response was already apparent by the end of the first postnatal week. Thus, a single NGF injection on PD2 was 5 times more effective than one on PD8 and there was little or no response on PD22. Of note, cholinergic neurochemical differentiation in caudate-putamen is delayed relative to that in the septum and adult levels of specific acitivity for ChAT are not achieved until PD25 (48). Thus, in these studies, a response to NGF was only registered in quite immature caudate-putamen neurons. The decreasing response of caudate-putamen to a single NGF injection may have been due to: (1) altered access of NGF administered via the intraventricular route to the caudate-putamen; (2) a decrease in NGF receptor binding, perhaps as a result of fewer NGF receptors; or (3) a true change in the response of these cholinergic eurons to NGF. Changes in the number of NGF receptors do not appear to explain the altered response of caudate-putamen neurons in older animals. Indeed, more, not less, high affinity NGF receptor binding is present in the caudate-putamen of mature animals (48) and this binding has been localized to the cell bodies of rather large $(22 \mu \mathrm{M}$ in diameter), presumably cholinergic, neurons (26). Altered access to administered NGF was also considered an unlikely explanation in view of the abrupt decrease in response over only 6 postnatal days (PD2 versus PD8). Evidence which directly addressed this consideration was provided by direct NGF injection in the caudate-putamen of PD22 animals. There was no change in ChAT activity as a result of this treatment. This result indicated that limited NGF access to caudate-putamen does not account for the lack of response. Of course, it is possible that exogenous NGF which enters caudateputamen is in some way prevented access to NGF receptors on cholinergic neurons. Regional differences in the access of exogenous NGF to its receptor could be responsible for the differential responsiveness of caudate-putamen and septum. However, current data do not preclude the possibility of a true change in the response of caudate- putamen neurons to NGF during development. In vitro studies may be required to decide this issue. Precedents for developmentally specific alterations in NGF responses have been described for NGF targets in the PNS (5). For example, though bovine adrenal medullary cells from fetal animals respond to NGF with increased neurotransmitter enzyme activity and neurite outgrowth, both responses are lost by adulthood. Moreover, this change occurs in spite of the continued presence of NGF receptors (33).

By reviewing the time-course studies in the context of other recent data, we can attempt to follow NGF action in the days following a single injection on PD2. CNS receptors which bind NGF with high affinity (26) and are physicochemically similar to those in the periphery (27) are present in early postnatal days $(24,48)$ and probably mediated NGF actions (12). If observations in adult animals serve as a guide (30), at least some fraction of NGF bound to hippocampal receptors was internalized and retrogradely transported to cholinergic cell bodies. The period which intervened between NGF injection and the septal response was at least 24 hours; from prior data (30) this lag period would allow ample time for retrograde transport to bring NGF from the hippocampus to septum. The event set in motion by internalized NGF molecules are as yet undefined. It is also not clear what contribution to NGF actions is made by molecules which bind directly to septal cell bodies. However, since at PD24 these cells are just beginning cholinergic neurochemical differentiation, it is likely that increased ChAT activity represented an NGF effect on the synthesis of new ChAT enzyme molecules in cholinergic cell bodies. It is probable that newly synthesized ChAT molecules were then transported anterogradely to hippocampus. The pronounced lag in hippocampal ChAT activity relative to septum is consistent with this suggestion. A preliminary calculation of the transport rate of ChAT molecules through the axon can be made on the basis of the delay between ChAT activity responses in the septum and hippocampus. Since the ChAT activity assayed in the hippocampus reflects the presence of ChAT molecules throughout its length, new ChAT molecules can be assumed to be distributed around the midpoint, or at $4 \mathrm{~mm}$, from the fornix. By subtracting the time of the septal ChAT activity peak from the timing of the initial hippocampal response, the velocity of anterograde transport of ChAT molecules can be estimated to be $2 \mathrm{~mm}$ per day. In agreement with earlier estimates for ChAT trans- 
port in peripheral nerve (49), this result suggests that ChAT molecules are moved by slow axoplasmic transport. These observations, and those given earlier, suggest that examination of NGF effects on forebrain cholinergic neurons will provide further insights regarding the dynamics of NGF action in the CNS.

\section{ACKNOWLEDGMENT}

The authors are grateful to Drs. Eric Shooter and Silvio Varon for their many contributions to research on NGF. William C. Mobley wishes to thank his mentor, Dr. Shooter, for unfailing friendship and support and for the encouragement to begin studies of NGF actions in the CNS.

This work was supported in part by USPHS grants N.S. 00603, N.S. 17642, N.S. 19613, N.S. 01015, N.S. 24054, BRSG grant SO7RR05355, and grants from the March Dimes Birth Defects Foundation. We thank Holley Arft for expert secretarial assistance and Carl Hildebrandt, Christopher Hudson and Julie Ong for excellent technical assistance.

\section{REFERENCES}

1. Berg, D. K. 1983. New neuronal growth factors. Annu, Rev. Neurosci. 7:149-170.

2. Varon, S., Manthorpe, M., and Williams, L. R. 1984. Neuronotrophic and neurite-promoting factors and their clinical potentials. Dev. Neurosci. 6:73-100.

3. Mobley, W. C., Server, A. C., Ishii, D. N., Riopelle, R. J., and Shooter, E. M. 1977. Nerve growth factor. N. Engl. J, Med. 297:1096-1104; 1149-1158; 1211-1218.

4. Greene, L. A., and Shooter, E. M. 1980. The nerve growth factor: biochemistry, synthesis, and mechanism of action. Annu. Rev. Neurosci. 3:353-402.

5. Thoenen, H., and Barde, Y. A. 1980. Physiology of nerve growth factor. Physiol. Rev. 60:1284-1335.

6. Thoenen, H., and Edgar, D. 1985. Neurotrophic factors. Science 229:238-242.

7. Honegger, P., and Lenoir, D. 1982. Nerve growth factor (NGF) stimulation of cholinergic telencephalic neurons in aggregating cell cultures. Dev. Brain Res. 3:229-238.

8. Gnahn, H., Hefti, F., Heumann, R., Schwab, M. E., and Thoenen, H. 1983. NGF-mediated increases of choline acetyltransferase (ChAT) in the neonatal rat forebrain: evidence for a physiological role of NGF in the brain? Dev. Brain Res. 9:45-52.

9. Hefti, F., Hartikka, F., Eckenstein, F., Gnahn, H. Heumann, R., and Schwab, M. 1985. Nerve growth factor increases choline acetyltransferase but not survival or fiber outgrowth of cultured fetal septal cholinergic neurons. Neuroscience 14:55-68.

10. Mobley, W. C., Rutkowski, J, L., Tennekoon, G. I., Buchanan, K., Johnston, M. V. Choline acetyltransferase activity in striatum of neonatal rats increased by nerve growth factor. Science 229:284-287.

11. Martinez, H. J., Dreyfus, C. F., Jonakait, F. R., and Black, I. B. 1985. Nerve growth factor promotes cholinergic development in brain striatal cultures. Proc. Natl. Acad. Sci. U.S.A. 82:7777-7781.
12. Mobley, W. C., Rutkowski, J. L., Tennekoon, G. I., Gemski, J., Buchanan, K., and Johnston, M. V. 1986. Nerve growth factor increases choline acetyltransferase activity in developing basal forebrain neurons. Mol. Brain Res. 1:53-62.

13. Wainer, B. H., Hsiang, J., Hoffman, P. C., Heller, A., and Mobley, W. C. 1986. Nerve growth factor enhances central cholinergic cell survival and fiber proliferation in reaggregate cultures. Neurosci. Lett. S17, 1986.

14. Johnston, M. V., McKinney, M., and Coyle, J. T. 1979. Evidence for a cholinergic projection to neocortex from neurons in basal forebrain. Proc. Natl. Acad. Sci. 76:5392-5396.

15. Coyle, J. T., Price, D. L., and DeLong, M. R. 1983. Alzheimer's disease: a disorder of cortical cholinergic innervation. Science 219:1184-1190.

16. Drachman, D. A. 1977. Memory and cognitive function in man: does the cholinergic system have a specific role? Neurology 27:783-790.

17. Schwarz, M., Ikonomidou, C., Klockgether, T., Turski, L., Ellenbroek, B., and Sontag, K.-H. 1986. The role of striatal cholinergic mechanisms for the development of limb rigidity: an electromyographic study in rats. Brain Res. 373:365-372.

18. Lalley, P. M., Rossi, G. V., and Baker, W. W. 1970. Analysis of local cholinergic tremor mechanisms following selective neurochemical lesions. Exp. Neurol. 27, 258-275.

19. Crutcher, K. A., and Collins, F. 1982 . In vitro evidence for two distinct hippocampal growth factors: basis of neuronal plasticity? Science 217:67-68.

20. Korsching, S., Auburgen, G., Heumann, R., Scott, J., and Thoenen H. 1985. Levels of nerve growth factor and its mRNA in the central nervous system of the rat correlate with cholinergic innervation. EMBO J. 4:1389-1393.

21. Whittemore, S. R., Ebendal, T., Larkfors, L., Olson, L., Seiger, A., Stromberg, I., and Persson, H. 1986. Developmental and regional expression of nerve growth factor messenger RNA and protein in the rat central nervous system. Proc. Natl. Acad. Sci. U.S.A. 83:817-821.

22. Shelton, D. S., and Reichardt, L. F. 1986. Studies on the expression of the nerve growth factor (NGF) gene in the central nervous system: level and regional distribution of NGF mRNA suggest that NGF functions as a trophic factor for several distinct populations of neurons. Proc. Natl. Acad. Sci. USA. 83:2714-2718.

23. Large, T. H., Bodary, S. C., Clegg, D. O., Weskamp, G., Otten, U., and Reichardt, L. F. 1986. Nerve growth factor gene expression in the developing rat brain. Science 234, $352-355$.

24. Frazier, W. A., Boyd, L. F., Szutowicz, A., Pulliam, M. W., and Bradshaw, R. A. 1974. Specific binding sites for ${ }^{125}$ Inerve growth factor in peripheral tissues and brain. Biochem. Biophys. Res. Commun. 57:1096-1103.

25. Zimmerman, A., Sutter, A., Samuelson, J., and Shooter, E. M. 1978. A serological assay for the detection of cell surface receptors of nerve growth factor. J. Supramol. Struct. 9:351361.

26. Richardson, P. M., Verge Issa, V. M. K., and Riopelle, R. J. 1986. Distribution of neuronal receptors for nerve growth factor in the rat. J. Neurosci. 6:2312-2321.

27. Taniuchi, M., Schweitzer, J. B., and Johnson, E. M. 1986. Nerve growth factor receptor molecules in rat brain. Proc. Natl. Acad. Sci. U.S.A. 83:1950-1954.

28. Springer, J. E., Koh, S., Tayrien, M. W., and Loy, R. 1986. Basal forebrain magnocellular neurons stain for nerve growth factor receptor: correlation with cholinergic cell bodies and effects of axotomy. J. Neurosci. Res., in press.

29. Hefti, F., Hartikka, J., Salvatierra, A., Weiner, W. J., and Mash, D. C. 1986. Localization of nerve growth factor receptors in cholinergic neurons of the human basal forebrain. Neurosci. Let. 69:37-41.

30. Schwab, M. E., Otten, U., Agid, Y., and Thoenen, H. 1979. 
Nerve growth factor (NGF) in the rat CNS: absence of specific retrograde axonal transport and tyrosine hydroxylase induction in locus coeruleus and substantia nigra. Brain Res. 168:473-483.

31. Seiler, M., and Schwab, M. E. 1984. Specific retrograde transport of nerve growth factor (NGF) from neocortex to nucleus basalis in the rat. Brain Res. 300:33-39.

32. Otten, U., Weskamp, G., Schlumpf, M., Lichtensteiger, W., and Mobley, W. C. 1985. Effects of antibodies against nerve growth factor on developing cholinergic forebrain neurons in the rat. Soc. Neurosci. Abstr. 11:661.

33. Naujoks, K. W., Korsching, S., Rohrer, H., and Thoenen, H. 1982. Nerve growth factor-mediated induction of tyrosine hydroxylase and of neurite outgrowth in cultures of bovine adrenal chromaffin cells: dependence on developmental stage. Dev. Biol. 92:365-379.

34. Otten, U. 1984. Nerve growth factor and the peptidergic sensory neurons. Trends Pharmacol. Sci. 5:307-310.

35. Mobley, W. C., Schenker, A., and Shooter, E. 1976. Characterization and isolation of proteolytically modified nerve growth factor. Biochemistry 15:5543-5552.

36. Levey, A. I., Armstrong, D. M., Atweh, S. F., Terry, R. D., and Wainer, B. H. 1983. Monoclonal antibodies to choline acetyltransferase: production, specificity and immunohistochemistry. J. Neurosci. 3:1-9.

37. Mesulam, M. M., Mufson, E. J., Wainer, B. H., and Levey, A. I. 1983. Central cholinergic pathways in the rat: an overview based on an alternative nomenclature (Ch1-Ch6). Neuroscience 10:1185-1201.

38. Bull, G., and Oderfeld-Nowak, B. 1971. Standardization of a radiochemical assay of cholinergic acetyltransferase. J. Neurochem. 18:935-947.

39. Bradford, M. 1976. A rapid and sensitive method for the quantitation of microgram quantities of protein utilizing the principle of protein dye binding. Anal. Biochem. 72:248-253.

40. Nadler, J. V., Matthews, D. A., Cotman, D. W., and Lynch, G. S. 1974. Development of cholinergic innervation in the hippocampal formation of the rat. II Quantitative changes in choline acetyltransferase and acetylcholinesterase activities. Dev. Biol. 36:142-154.

41. Shelton, D. L., Nadler, J. V., Cotman, C. W. 1979. Development of high affinity choline uptake an associated acetylcholine synthesis in the rat fascia dentata. Brain Res. 163:263-275.

42. Milner, T. A., Loy, R., Amaral, D. G. 1983. An anatomical study of the development of the septohippocampal projection in the rat. Dev. Brain. Res. 8:343-371.

43. Bjerre, B., Bjorklund, A., Mobley, W., and Rosengren, E. 1975. Short- and long-term effects of nerve growth factor on the sympathetic nervous system in the adult mouse. Brain Res. 94:263-277.

44. Hefti, F., Dravid, A., and Hartikka, J. 1984. Chronic intraventricular injections of nerve growth factor elevate hippocampal choline acetyltransferase activity in adult rats with partial septo-hippocampal lesions. Brain Res. 293:305311.

45. Williams, L. R., Varon, S., Peterson, G. M., Wictorin, K., Fischer, W., Bjorklund, A., and Gaye, F. H. 1986. Continvous infusion of nerve growth factor prevents basal forebrain neuronal death after fimbria-fornix transection. Proc. Natl. Acad. Sci. U.S.A. 83:9231-9235.

46. Hefti, F., 1986. Nerve growth factor promotes survival of septal cholinergic neurons after fimbrial transections. J. Neurosci. 6:2155-2162.

47. Kromer, L. F. 1987. Nerve growth factor treatment after brain injury prevents neuronal death. Science 235:214-216.

48. Mobley, W. C., Edwards, R. H., Riopelle, R. J., Otten, U. H., and Johnston, M. V. 1986. Nerve growth factor (NGF) in the corpus striatum: the developmental expression of NGF and its receptor. Soc. Neurosci. Abstr. 12:393.

49. Wooten, G. F., and Cheng, C. H. 1980. Transport and turnover acetylcholinesterase and choline acetyltransferase in rat sciatic nerve and skeletal muscle. J. Neurochem. 34:359366. 